\title{
Correction to: The tree that hides the forest: cryptic diversity and phylogenetic relationships in the Palaearctic vector Obsoletus/Scoticus complex (Diptera: Ceratopogonidae) at the European level
}

Antoine Mignotte ${ }^{1,2^{*}}$, Claire Garros ${ }^{1,2^{*}}$, Laetitia Gardès ${ }^{1,3}$, Thomas Balenghien ${ }^{1,2,4}$, Maxime Duhayon ${ }^{1,2}$, Ignace Rakotoarivony ${ }^{1,2}$, Laura Tabourin 1,2, Léa Poujol 1,2, Bruno Mathieu ${ }^{5}$, Adolfo Ibañez-Justicia ${ }^{6}$, Ahmet Deniz ${ }^{7}$, Aleksandar Cvetkovikj ${ }^{8}$, Bethan V. Purse ${ }^{9}$, David W. Ramilo ${ }^{10}$, Despoina Stougiou ${ }^{11}$, Doreen Werner ${ }^{12}$, Dubravka Pudar ${ }^{13}$, Dušan Petrić ${ }^{13}$, Eva Veronesi $^{14}$, Frans Jacobs $^{6}$, Helge Kampen ${ }^{15}$, Isabel Pereira da Fonseca ${ }^{10}$, Javier Lucientes ${ }^{16}$, Javier Navarro ${ }^{17}$, Josue Martinez-de la Puente ${ }^{18,19}$, Jovana Stefanovska ${ }^{8}$, Kate R. Searle ${ }^{20}$, Khalid Khallaayoune ${ }^{4}$, C. Lorna Culverwell ${ }^{18,21}$, Magdalena Larska ${ }^{22}$, Maria Bourquia ${ }^{2,4}$, Maria Goffredo ${ }^{23}$, Marina Bisia ${ }^{11}$, Marion England ${ }^{24}$, Matthew Robin ${ }^{25}$, Michela Quaglia ${ }^{23}$, Miguel Ángel Miranda-Chueca ${ }^{26}$, René Bødker ${ }^{27}$, Rosa Estrada-Peña ${ }^{16}$, Simon Carpenter ${ }^{24}$, Simona Tchakarova ${ }^{28}$, Sofia Boutsini ${ }^{11}$, Ståle Sviland ${ }^{29}$, Stefanie M. Schäfer ${ }^{9}$, Zanda Ozolin,a ${ }^{30}$, Zanda Seglin, ${ }^{30}$, Zati Vatansever ${ }^{31}$ and Karine Huber ${ }^{1}$

\section{Correction to: Parasites Vectors (2020) 13:265}

https://doi.org/10.1186/s13071-020-04114-1

Following publication of the original article [1], the authors flagged that unfortunately there is an error with the affiliations.

The affiliation of Zati Vatansever (31) is listed as "Veterinary Control Central Research Institute, Ankara, Turkey".

However, the correct affiliation is "Faculty of Veterinary Medicine, Department of Parasitology, Kafkas University, Kars, Turkey".

The authors apologize for the inconvenience caused.

The original article can be found online at https://doi.org/10.1186/s1307 1-020-04114-1.

*Correspondence: antoine.mignotte@cirad.fr; claire.garros@cirad.fr

${ }^{1}$ ASTRE, Univ Montpellier, Cirad, INRAE, Montpellier, France

Full list of author information is available at the end of the article

\section{Author details}

${ }^{1}$ ASTRE, Univ Montpellier, Cirad, INRAE, Montpellier, France. ${ }^{2}$ Cirad, UMR ASTRE, Montpellier F-34398, France. ${ }^{3}$ Cirad, UMR ASTRE, Petit-Bourg, F97170 Guadeloupe, France. ${ }^{4}$ Unité Parasitologie et Maladies Parasitaires, Institut Agronomique et Vétérinaire Hassan II, Rabat 10100, Morocco.

${ }^{5}$ Institute of Parasitology and Tropical Pathology of Strasbourg, Université de Strasbourg, DIHP UR 7292, Strasbourg F-67000, France. ${ }^{6}$ Centre for Monitoring of Vectors, National Reference Centre, Netherlands Food and Consumer Product Safety Authority, Wageningen, The Netherlands. ${ }^{7}$ Veterinary Control Central Research Institute, Ankara, Turkey. ${ }^{8}$ Department of Parasitology and Parasitic Diseases, Faculty of Veterinary Medicine, Ss. Cyril and Methodius University, Skopje, Republic of North Macedonia. ${ }^{9}$ Centre for Ecology and Hydrology, UK Centre for Ecology and Hydrology, Wallingford OX10 8BB, UK. ${ }^{10} \mathrm{CIISA}$ - Centro de Investigação Interdisciplinar em Sanidade Animal, Faculdade de Medicina Veterinária, Universidade de Lisboa, Avenida da Universidade Técnica, Lisboa 1300-477, Portugal. ${ }^{11}$ Veterinary Centre of Athens Department of Parasitology-Parasitic Diseases, Entomology \& Bee Health, Athens, Greece. ${ }^{12}$ Leibniz-Centre for Agricultural Landscape Research, Müncheberg, Germany. ${ }^{13}$ Faculty of Agriculture, University of Novi Sad, Novi Sad, Serbia. ${ }^{14}$ National Centre for Vector Entomology, Institute of Parasitology, University of Zürich, Zürich, Switzerland. ${ }^{15}$ Friedrich-Loeffler-Institut, Federal Research Institute for Animal Health, Greifswald, Germany. ${ }^{16}$ Department of Animal Pathology, AgriFood Institute of Aragón (IA2) Veterinary Faculty, Zaragoza 50013, Spain. ${ }^{17}$ Laboratorio de Producción y Sanidad Animal de Granada, Departamento de Microbiología, Junta de Andalucía, Granada, Spain. ${ }^{18}$ Doñana Biological Station, CSIC, Sevilla, Spain. ${ }^{19}$ Centro de Investigación Biomédica en Red de Epidemiología y Salud Pública (CIBERESP), Madrid, Spain.

(c) The Author(s) 2020. This article is licensed under a Creative Commons Attribution 4.0 International License, which permits use, sharing, adaptation, distribution and reproduction in any medium or format, as long as you give appropriate credit to the original author(s) and the source, provide a link to the Creative Commons licence, and indicate if changes were made. The images or other third party material in this article are included in the article's Creative Commons licence, unless indicated otherwise in a credit line to the material. If material is not included in the article's Creative Commons licence and your intended use is not permitted by statutory regulation or exceeds the permitted use, you will need to obtain permission directly from the copyright holder. To view a copy of this licence, visit http://creativecommons.org/licenses/by/4.0/. The Creative Commons Public Domain Dedication waiver (http://creativecommons.org/publicdomain/zero/1.0/) applies to the data made available in this article, unless otherwise stated in a credit line to the data. 
${ }^{20}$ Centre for Ecology \& Hydrology, Edinburgh OX10 8BB, UK. ${ }^{21}$ Department of Virology, University of Helsinki, Medicum, Haartmaninkatu 3, Helsinki 00014, Finland. ${ }^{22}$ National Veterinary Research Institute, Puławy, Poland. ${ }^{23}$ Istituto Zooprofilattico Sperimentale dell'Abruzzo e del Molise 'G. Caporale', Campo Boario, 64100 Teramo, Italy. ${ }^{24}$ The Pirbright Institute, Pirbright, UK. ${ }^{25}$ Department of Epidemiology and Population Health, Institute of Infection and Global Health, University of Liverpool, Leahurst, Chester High Road, Neston, Cheshire, Leahurst CH64 7TE, UK. ${ }^{26}$ Applied Zoology and Animal Conservation Research Group, University of the Balearic Islands UIB, Palma, Spain. ${ }^{27}$ University

of Copenhagen, Copenhagen, Denmark. ${ }^{28}$ National Diagnostic and Research Veterinary Medical Institute, Sofia, Bulgaria. ${ }^{29}$ Norwegian Veterinary Institute, Oslo, Norway. ${ }^{30}$ Institute of Food safety, Animal Health and Environment 'BIOR', Riga, Latvia. ${ }^{31}$ Faculty of Veterinary Medicine, Department of Parasitology, Kafkas University, Kars, Turkey.

Published online: 22 September 2020

\section{Reference}

1. Mignotte A, Garros C, Gardès L, et al. The tree that hides the forest: cryptic diversity and phylogenetic relationships in the Palaearctic vector Obsoletus/Scoticus Complex (Diptera: Ceratopogonidae) at the European level. Parasites Vectors. 2020;13:265. https://doi.org/10.1186/s13071-020-04114 $-1$.

\section{Publisher's Note}

Springer Nature remains neutral with regard to jurisdictional claims in published maps and institutional affiliations. 